$£$ million Balance of Payments basis seasonally adjusted

\begin{tabular}{|c|c|c|c|c|c|c|c|c|c|}
\hline & \multicolumn{3}{|c|}{ Total trade in goods } & \multicolumn{3}{|c|}{ Total excluding oil } & \multicolumn{3}{|c|}{$\begin{array}{l}\text { Total excluding } \\
\text { oil and erratics }\end{array}$} \\
\hline & Exports & Imports & Balance & Exports & Imports & Balance & Exports & Imports & Balance \\
\hline & BOKG & BOKH & BOKI & ELBM & ENXP & BQKH & BPBL & BQBG & BPAP \\
\hline 2003 & 188320 & 236927 & -48607 & 173712 & 225695 & -51983 & 161431 & 212703 & -51272 \\
\hline 2004 & 190874 & 251774 & -60900 & 174674 & 236467 & -61793 & 162463 & 224255 & -61792 \\
\hline 2005 & 211608 & 280197 & -68589 & 191814 & 258208 & -66394 & 179356 & 245199 & -65843 \\
\hline 2006 & 243633 & 319945 & -76312 & 220460 & 293978 & -73518 & 208146 & 278578 & -70432 \\
\hline 2007 & 220858 & 310612 & -89754 & 198102 & 283825 & -85723 & 185038 & 269470 & -84432 \\
\hline 2008 & 251643 & 345024 & -93381 & 219498 & 307011 & -87513 & 204398 & 291061 & -86663 \\
\hline 2004 Q3 & 48647 & 63840 & -15193 & 44443 & 59792 & -15349 & 41197 & 56620 & -15423 \\
\hline Q4 & 49953 & 66062 & -16109 & 45382 & 61598 & -16216 & 42277 & 58594 & -16317 \\
\hline 2005 Q1 & 48808 & 65179 & -16371 & 44398 & 60880 & -16482 & 41223 & 57673 & -16450 \\
\hline Q2 & 51357 & 67239 & -15882 & 46591 & 62393 & -15802 & 43728 & 59370 & -15642 \\
\hline Q3 & 54399 & 72354 & -17955 & 49125 & 65700 & -16575 & 45914 & 62322 & -16408 \\
\hline Q4 & 57044 & 75425 & -18381 & 51700 & 69235 & -17535 & 48491 & 65834 & -17343 \\
\hline 2006 Q1 & 65698 & 83660 & -17962 & 60063 & 77138 & -17075 & 57128 & 73396 & -16268 \\
\hline Q2 & 67986 & 85925 & -17939 & 61500 & 79306 & -17806 & 58165 & 75408 & -17243 \\
\hline Q3 & 55821 & 75643 & -19822 & 49714 & 68560 & -18846 & 47015 & 64696 & -17681 \\
\hline Q4 & 54128 & 74717 & -20589 & 49183 & 68974 & -19791 & 45838 & 65078 & -19240 \\
\hline 2007 Q1 & 53516 & 75133 & -21617 & 48740 & 69558 & -20818 & 45681 & 65627 & -19946 \\
\hline Q2 & 54580 & 74985 & -20405 & 48970 & 69063 & -20093 & 45235 & 65531 & -20296 \\
\hline Q3 & 55524 & 79290 & -23766 & 49754 & 72171 & -22417 & 46715 & 68477 & -21762 \\
\hline Q4 & 57238 & 81204 & -23966 & 50638 & 73033 & -22395 & 47407 & 69835 & -22428 \\
\hline 2008 Q1 & 60636 & 84162 & -23526 & 53454 & 75638 & -22184 & 50162 & 71978 & -21816 \\
\hline Q2 & 64980 & 88666 & -23686 & 55813 & 77957 & -22144 & 52184 & 73930 & -21746 \\
\hline Q3 & 65944 & 89803 & -23859 & 56870 & 78765 & -21895 & 52632 & 74552 & -21920 \\
\hline Q4 & 60083 & 82393 & -22310 & 53361 & 74651 & -21290 & 49420 & 70601 & -21181 \\
\hline 2009 Q1 & 55799 & 76654 & -20855 & 50352 & 70410 & -20058 & 47212 & 66573 & -19361 \\
\hline Q2 & 54841 & 74583 & -19742 & 48961 & 68371 & -19410 & 45706 & 64002 & -18296 \\
\hline Q3 & 56910 & 76589 & -19679 & 50909 & 69130 & -18221 & 47789 & 65077 & -17288 \\
\hline 2006 Nov & 18257 & 25076 & -6819 & 16614 & 23045 & -6431 & 15421 & 21743 & -6322 \\
\hline Dec & 17750 & 24953 & -7203 & 16203 & 23215 & -7012 & 15189 & 21862 & -6673 \\
\hline 2007 Jan & 17960 & 24737 & -6777 & 16432 & 22788 & -6356 & 15378 & 21633 & -6255 \\
\hline Feb & 17514 & 24719 & -7205 & 15909 & 23105 & -7196 & 14966 & 21655 & -6689 \\
\hline Mar & 18042 & 25677 & -7635 & 16399 & 23665 & -7266 & 15337 & 22339 & -7002 \\
\hline Apr & 18069 & 24578 & -6509 & 16173 & 22532 & -6359 & 14507 & 21472 & -6965 \\
\hline May & 18078 & 24965 & -6887 & 16313 & 23090 & -6777 & 15254 & 21793 & -6539 \\
\hline Jun & 18433 & 25442 & -7009 & 16484 & 23441 & -6957 & 15474 & 22266 & -6792 \\
\hline Jul & 18426 & 26275 & -7849 & 16415 & 24020 & -7605 & 15297 & 22775 & -7478 \\
\hline Aug & 18653 & 26286 & -7633 & 16983 & 23978 & -6995 & 15968 & 22767 & -6799 \\
\hline Sep & 18445 & 26729 & -8284 & 16356 & 24173 & -7817 & 15450 & 22935 & -7485 \\
\hline Oct & 18916 & 26878 & -7962 & 16815 & 24230 & -7415 & 15670 & 23066 & -7396 \\
\hline Nov & 19517 & 27565 & -8048 & 17370 & 24608 & -7238 & 16307 & 23446 & -7139 \\
\hline Dec & 18805 & 26761 & -7956 & 16453 & 24195 & -7742 & 15430 & 23323 & -7893 \\
\hline 2008 Jan & 20007 & 27956 & -7949 & 17743 & 25096 & -7353 & 16706 & 23924 & -7218 \\
\hline Feb & 20326 & 28202 & -7876 & 17903 & 25535 & -7632 & 16729 & 24011 & -7282 \\
\hline Mar & 20303 & 28004 & -7701 & 17808 & 25007 & -7199 & 16727 & 24043 & -7316 \\
\hline Apr & 21205 & 28912 & -7707 & 18522 & 25836 & -7314 & 17337 & 24403 & -7066 \\
\hline May & 21590 & 29360 & -7770 & 18559 & 25846 & -7287 & 17347 & 24550 & -7203 \\
\hline Jun & 22185 & 30394 & -8209 & 18732 & 26275 & -7543 & 17500 & 24977 & -7477 \\
\hline Jul & 22739 & 30980 & -8241 & 19721 & 26318 & -6597 & 18166 & 24947 & -6781 \\
\hline Aug & 21442 & 29591 & -8149 & 18527 & 26384 & -7857 & 17215 & 24878 & -7663 \\
\hline Sep & 21763 & 29232 & -7469 & 18622 & 26063 & -7441 & 17251 & 24727 & -7476 \\
\hline Oct & 21036 & 28458 & -7422 & 18543 & 25425 & -6882 & 17130 & 23972 & -6842 \\
\hline Nov & 19658 & 27404 & -7746 & 17353 & 24908 & -7555 & 15953 & 23571 & -7618 \\
\hline Dec & 19389 & 26531 & -7142 & 17465 & 24318 & -6853 & 16337 & 23058 & -6721 \\
\hline 2009 Jan & 18425 & 25997 & -7572 & 16527 & 23901 & -7374 & 15591 & 22468 & -6877 \\
\hline Feb & 18790 & 25560 & -6770 & 17094 & 23738 & -6644 & 16104 & 22565 & -6461 \\
\hline Mar & 18584 & 25097 & -6513 & 16731 & 22771 & -6040 & 15517 & 21540 & -6023 \\
\hline Apr & 18612 & 25620 & -7008 & 16713 & 23624 & -6911 & 15626 & 22135 & -6509 \\
\hline May & 17995 & 24294 & -6299 & 16024 & 22402 & -6378 & 14974 & 20918 & -5944 \\
\hline Jun & 18234 & 24669 & -6435 & 16224 & 22345 & -6121 & 15106 & 20949 & -5843 \\
\hline Jul & 18830 & 25390 & -6560 & 16758 & 22825 & -6067 & 15701 & 21255 & -5554 \\
\hline Aug & 18627 & 24807 & -6180 & 16697 & 22446 & -5749 & 15815 & 21390 & -5575 \\
\hline Sep & 19453 & 26392 & -6939 & 17454 & 23859 & -6405 & 16273 & 22432 & -6159 \\
\hline Oct & $20173^{\dagger}$ & $27189^{\dagger}$ & $-7016^{\dagger}$ & $17932^{\dagger}$ & $24733^{\dagger}$ & $-6801^{\dagger}$ & $16872^{\dagger}$ & $23231^{\dagger}$ & $-6359^{\dagger}$ \\
\hline Nov & 20194 & 26978 & -6784 & 17903 & 24356 & -6453 & 16835 & 22954 & -6119 \\
\hline
\end{tabular}


Indices 2005=100 Balance of Payments basis

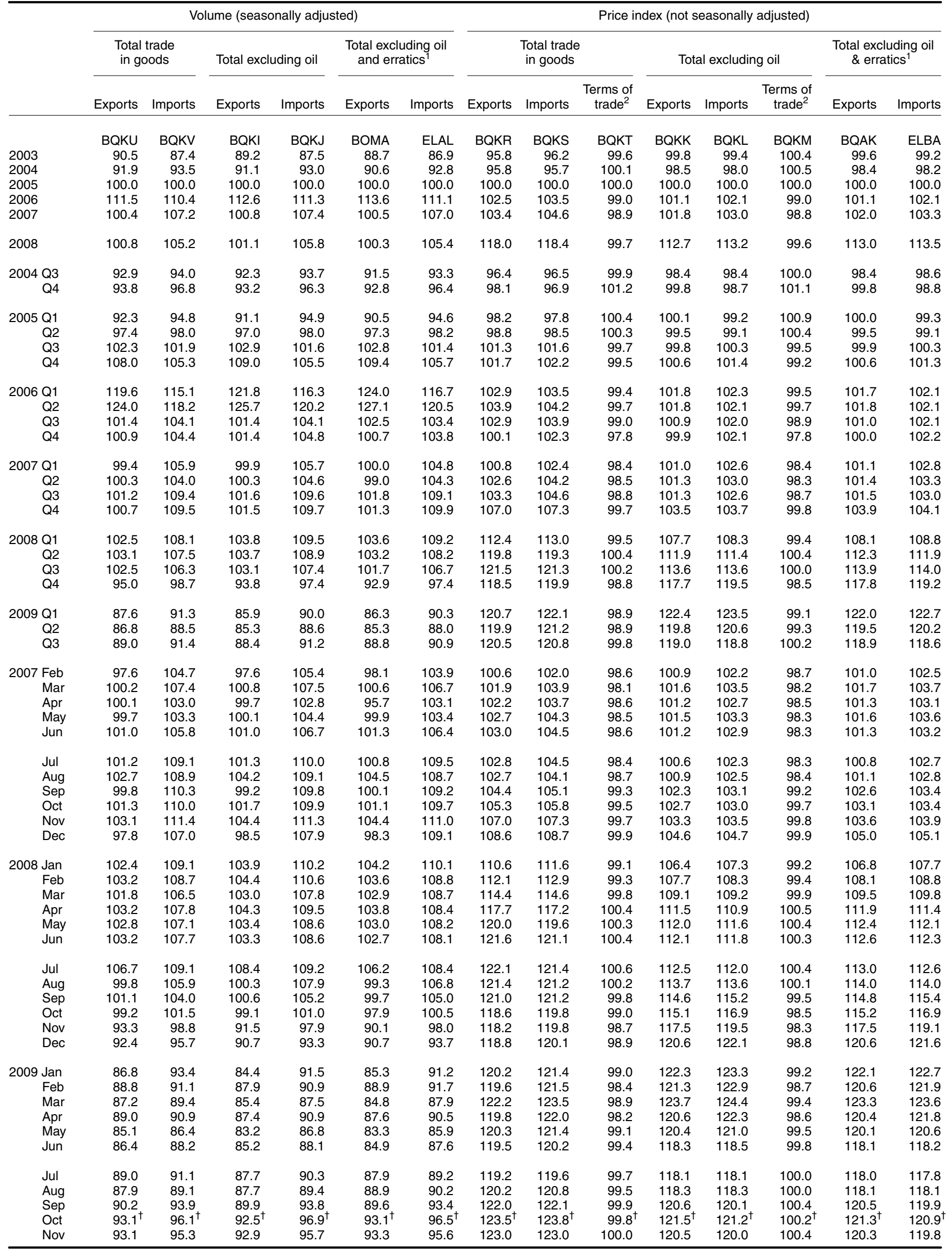


15.3 United Kingdon tratei in goods, by commoditty group'

$£$ million Balance of Payments basis seasonally adjusted

\begin{tabular}{|c|c|c|c|c|c|c|c|c|c|c|c|c|c|c|c|}
\hline & \multicolumn{3}{|c|}{$\begin{array}{l}\text { Food, beverages and } \\
\text { tobacco }(\text { SITC } 0+1)\end{array}$} & \multicolumn{3}{|c|}{$\begin{array}{l}\text { Basic materials } \\
\quad(\text { SITC 2+4) }\end{array}$} & \multicolumn{3}{|c|}{ Fuels (SITC 3) } & \multicolumn{3}{|c|}{$\begin{array}{l}\text { Semi-manufactures } \\
\text { (SITC 5+6) }\end{array}$} & \multicolumn{3}{|c|}{$\begin{array}{l}\text { Finished manufactures } \\
\text { (SITC 7+8) }\end{array}$} \\
\hline & Exports & Imports & Balance & Exports & Imports & Balance & Exports & Imports & Balance & Exports & Imports & Balance & Exports & Imports & Balance \\
\hline & BOPL & BQAR & ELBE & BOPM & BQAS & ELBF & BOPN & BQAT & ELBG & BOPO & BQAU & ELBH & BOPP & BQAV & ELBI \\
\hline 2003 & 10879 & 21187 & -10308 & 3335 & 6139 & -2804 & 16558 & 12311 & 4247 & 54492 & 56045 & -1553 & 102193 & 139641 & -37448 \\
\hline 2004 & 10577 & 22150 & -11573 & 3770 & 6338 & -2568 & 17885 & 17547 & 338 & 56467 & 60228 & -3761 & 101293 & 143704 & -42411 \\
\hline 2005 & 10647 & 23695 & -13048 & 3981 & 6770 & -2789 & 21496 & 25921 & -4425 & 59880 & 62677 & -2797 & 114484 & 159293 & -44809 \\
\hline 2006 & 10945 & 25013 & -14068 & 4892 & 7887 & -2995 & 25301 & 30888 & -5587 & 64843 & 69342 & -4499 & 136366 & 184745 & -48379 \\
\hline 2007 & 11769 & 26747 & -14978 & 5523 & 9561 & -4038 & 24700 & 31928 & -7228 & 68269 & 74437 & -6168 & 109408 & 165665 & -56257 \\
\hline 2008 & 13708 & 31118 & -17410 & 6626 & 10994 & -4368 & 35684 & 48578 & -12894 & 76179 & 79838 & -3659 & 117657 & 171930 & -54273 \\
\hline 2004 Q3 & 2601 & 5572 & -2971 & 991 & & -591 & 4734 & 4697 & 37 & 14289 & & -1131 & 25824 & & -10320 \\
\hline Q4 & 2685 & 5656 & -2971 & 991 & 1618 & -627 & 4954 & 5175 & -221 & 14678 & 15818 & -1140 & 26378 & 37306 & -10928 \\
\hline 2005 Q1 & 2651 & 5789 & -3138 & 984 & 1614 & -630 & 4766 & 5088 & -322 & 14713 & 15678 & -965 & 25477 & 36568 & -11091 \\
\hline Q2 & 2720 & 5939 & -3219 & 949 & 1651 & -702 & 5282 & 5536 & -254 & 14584 & 15460 & -876 & 27614 & 38152 & -10538 \\
\hline Q3 & 2643 & 5917 & -3274 & 1021 & 1734 & -713 & 5705 & 7555 & -1850 & 15183 & 15472 & -289 & 29566 & 41252 & -11686 \\
\hline 2006 Q1 & 2644 & 6196 & -3552 & 1112 & 1876 & -764 & 6129 & 8187 & -2058 & 15805 & 16730 & -925 & 39631 & 50125 & -10494 \\
\hline Q2 & 2715 & 27 & -3412 & 1184 & & -692 & 093 & & -510 & 264 & & -757 & 40418 & & -12371 \\
\hline Q3 & 2736 & 6256 & -3520 & 1205 & 18 & -692 & 6733 & 8237 & -1504 & 16047 & 18 & -1271 & 28793 & 472 & -12679 \\
\hline Q4 & 2850 & 6434 & -3584 & 1391 & 2238 & -847 & 5346 & 6861 & -1515 & 16727 & 18273 & -1546 & 27524 & 40359 & -12835 \\
\hline 2007 Q1 & 2912 & 6441 & -3529 & 1312 & & -881 & 5094 & & -1734 & & & -1232 & 26874 & & -14090 \\
\hline Q2 & 2881 & 654 & -3665 & 1440 & 239 & -952 & 6105 & 6892 & -787 & 16933 & 18 & -1479 & 26969 & & -13230 \\
\hline Q3 & 2969 & 6791 & -3822 & 1286 & 2495 & -1209 & 6229 & 8227 & -1998 & 17268 & 19131 & -1863 & 27504 & 42113 & -14609 \\
\hline Q4 & 3007 & 6969 & -3962 & 1485 & 2481 & -996 & 7272 & 9981 & -2709 & 17121 & 18715 & -1594 & 28061 & 42389 & -14328 \\
\hline 2008 Q1 & 3280 & 7412 & -4132 & 1553 & 2844 & -1291 & 7792 & & -3016 & 18373 & & -1141 & 29278 & 42961 & -13683 \\
\hline Q2 & 3363 & 7777 & -4414 & 1937 & & -939 & 10108 & & -2964 & 416 & & -983 & 29736 & & -14211 \\
\hline Q3 & 3572 & 7875 & -4303 & 1842 & 28 & -1053 & 10086 & & -3789 & 9840 & & -983 & 30083 & 791 & -13708 \\
\hline Q4 & 3493 & 8054 & -4561 & 1294 & 2379 & -1085 & 7698 & 10823 & -3125 & 18550 & 19102 & -552 & 28560 & 41231 & -12671 \\
\hline 2009 Q1 & 52 & 8077 & -4525 & 1155 & & -790 & 241 & & 565 & 767 & & -916 & 26635 & & -11900 \\
\hline Q2 & & 168 & -4534 & 1244 & & -598 & 493 & & -1271 & 17102 & & -1369 & 25924 & & -11784 \\
\hline Q3 & 3608 & 7876 & -4268 & 1330 & 1885 & -555 & 6425 & 8750 & -2325 & 18273 & 18069 & 204 & 26765 & 39333 & -12568 \\
\hline 2007 Jan & 964 & 2179 & -1215 & 412 & 679 & -267 & 1648 & 2354 & -706 & 5728 & 5952 & -224 & 9074 & & -4314 \\
\hline Feb & 968 & 2127 & -1159 & 456 & 736 & -280 & 1692 & & -341 & 5459 & 6038 & -579 & 8817 & & -4799 \\
\hline Mar & 980 & 2135 & -1155 & 444 & 778 & -334 & 1754 & 2441 & -687 & 5760 & 6189 & -429 & 8983 & 960 & -4977 \\
\hline Apr & 931 & 195 & -1264 & 471 & 786 & -315 & 063 & 2475 & -412 & 469 & 58 & -427 & 9049 & 3025 & -3976 \\
\hline May & 1003 & 210 & -1207 & 93 & 790 & -297 & 964 & 2147 & -183 & 800 & 6208 & -408 & 8735 & 3441 & -4706 \\
\hline Jun & 947 & 2141 & -1194 & 476 & 816 & -340 & 2078 & 2270 & -192 & 5664 & 6308 & -644 & 9185 & 13733 & -4548 \\
\hline Jul & 990 & & -1212 & 452 & & -396 & & & -407 & 636 & & -902 & 9051 & & -4838 \\
\hline & 1009 & & -1296 & 420 & & -430 & & & -858 & 5888 & & & 9439 & & -4561 \\
\hline Sep & 970 & 2284 & -1314 & 414 & & -383 & 2214 & 2947 & -733 & 5744 & 6300 & -556 & 9014 & 14224 & -5210 \\
\hline Oct & 988 & 2357 & -1369 & 460 & 819 & -359 & 2344 & 3121 & -777 & 5763 & 6208 & -445 & 9270 & 14147 & -4877 \\
\hline Nov & 1032 & 2335 & -1303 & 552 & 827 & -275 & 2384 & 3548 & -1164 & 5880 & 6259 & -379 & 9563 & 14376 & -4813 \\
\hline Dec & 987 & 2277 & -1290 & 473 & 835 & -362 & 2544 & 3312 & -768 & 5478 & 6248 & -770 & 9228 & 13866 & -4638 \\
\hline 2008 Jan & 129 & 2432 & -1303 & 452 & & -507 & 497 & & -1152 & 6150 & & -105 & 9673 & & -4777 \\
\hline Feb & 083 & 454 & -1371 & 71 & 1003 & -432 & 600 & & -732 & 6205 & & -381 & 9747 & & -4890 \\
\hline Mar & 068 & 2526 & -1458 & 530 & 882 & -352 & 2695 & 3827 & -1132 & 6018 & & -655 & 9858 & 13874 & -4016 \\
\hline Apr & 122 & 529 & -1407 & 624 & 873 & -249 & 931 & 3917 & -986 & 6253 & & -434 & 10129 & & -4574 \\
\hline May & 1122 & 579 & -1457 & 680 & 979 & -299 & 3388 & & -883 & 6523 & & -229 & 9750 & & -4840 \\
\hline Jun & 1119 & 2669 & -1550 & 633 & 1024 & -391 & 3789 & 4884 & -1095 & 6640 & 6960 & -320 & 9857 & 14654 & -4797 \\
\hline Jul & 1197 & & -1466 & 07 & & -287 & & & -2138 & & & & 10465 & & -4218 \\
\hline Aug & 1168 & 2590 & -1422 & 558 & & -395 & 3244 & & -1213 & 6521 & & -340 & 9791 & & -4743 \\
\hline Sep & 1207 & 2622 & -1415 & 577 & 948 & -371 & 3485 & 3923 & -438 & 6470 & 6998 & -528 & 9827 & 14574 & -4747 \\
\hline Oct & 1168 & 2655 & -1487 & 513 & 833 & -320 & 2911 & 4052 & -1141 & 6558 & 6614 & -56 & 9708 & 083 & -4375 \\
\hline Nov & 1118 & 2740 & -1622 & 377 & 803 & -426 & 2592 & 3548 & -956 & 6007 & 6323 & -316 & 9415 & 13730 & -4315 \\
\hline Dec & 1207 & 2659 & -1452 & 404 & 743 & -339 & 2195 & 3223 & -1028 & 5985 & 6165 & -180 & 9437 & 13418 & -3981 \\
\hline 2009 Jan & & & -1455 & 330 & & -320 & & & -853 & 5825 & & -450 & 8664 & & -4416 \\
\hline Feb & 1182 & & -1564 & 07 & & & & & -703 & 6203 & & & 8952 & & -4099 \\
\hline Mar & 1176 & 2682 & -1506 & 418 & 644 & -226 & 2060 & 3069 & -1009 & 5739 & & & 9019 & & -3385 \\
\hline Apr & 1219 & 2801 & -1582 & 482 & & -150 & 2123 & 2686 & -563 & 5937 & 6452 & -515 & 8703 & & -4127 \\
\hline May & 1201 & 2699 & -1498 & 370 & 621 & -251 & 2170 & 2391 & -221 & 5495 & 6160 & -665 & 8619 & 12221 & -3602 \\
\hline Jun & 1214 & 2668 & -1454 & 392 & 589 & -197 & 2200 & 2687 & -487 & 5670 & 5859 & -189 & 8602 & 12657 & -4055 \\
\hline Jul & & & -1440 & 413 & & -180 & & & -780 & & & -110 & 8926 & & -3997 \\
\hline & 1202 & 2634 & -1432 & 424 & 566 & -142 & & & -769 & 6088 & & 367 & 8696 & & -4163 \\
\hline Sep & 1244 & 2640 & -1396 & 493 & 726 & -233 & 2144 & 2920 & -776 & 6254 & 6307 & -53 & 9143 & 13551 & -4408 \\
\hline Oct & $1228^{\dagger}$ & $2775^{\dagger}$ & $-1547^{\dagger}$ & $523^{\dagger}$ & 666 & $-143^{\dagger}$ & $2428^{\dagger}$ & $2838^{\dagger}$ & $-410^{\dagger}$ & $6306^{\dagger}$ & $6721^{\dagger}$ & $-415^{\dagger}$ & $9507^{\dagger}$ & $13906^{\dagger}$ & $-4399^{\dagger}$ \\
\hline
\end{tabular}

1 More commodity detail is available on a seasonally adjusted Balance of

Payments basis in tables B1 to B11 inclusive, and C1 to C4 inclusive, of the

Source: Office for National Statistics: 01633456294

Monthly Review of External Trade Statistics. 
15.4 Volume index numbers, by commodity yroup'

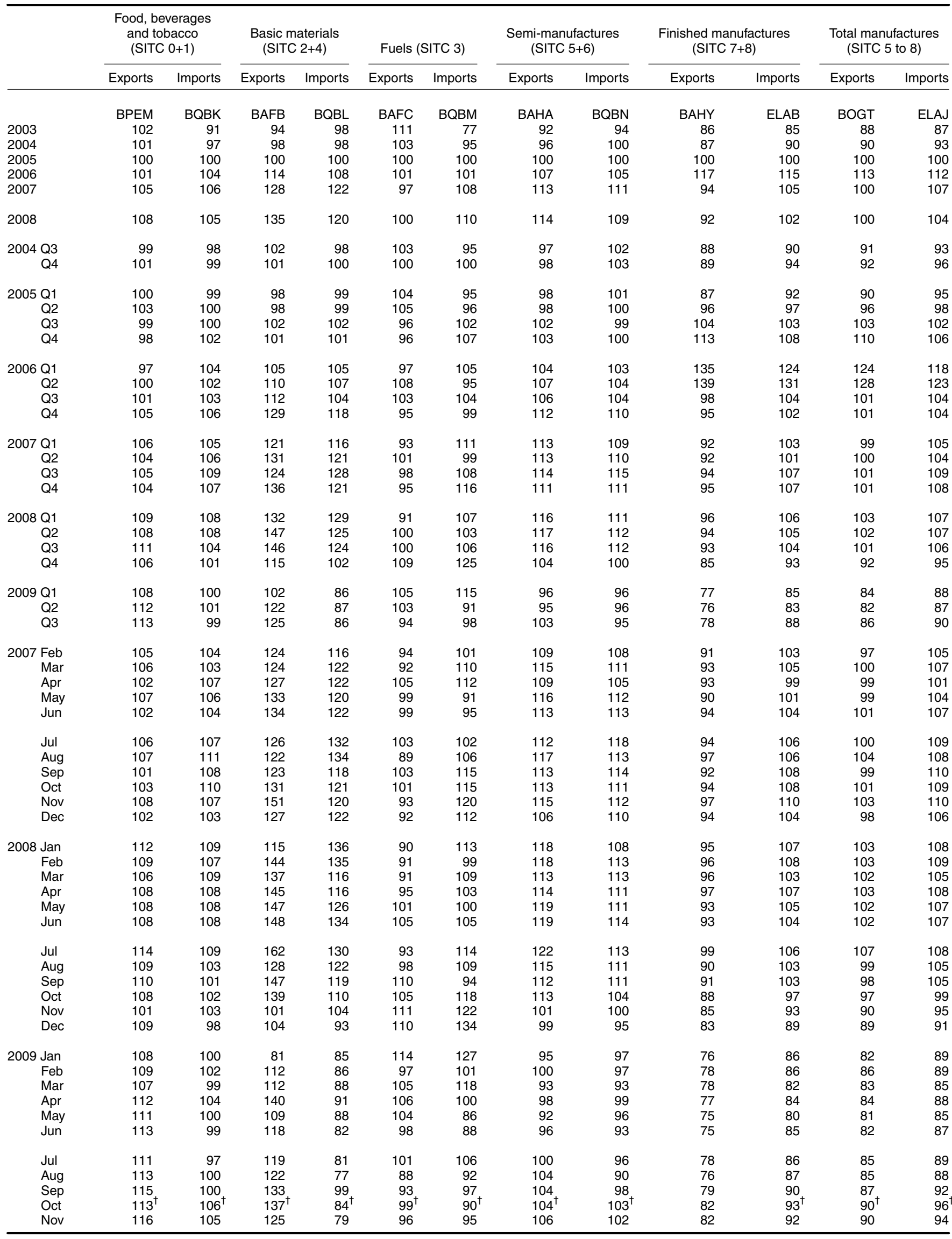

1 Commodity volumes are shown in more detail on a seasonally adjusted

Source: Office for National Statistics: 01633456294 Balance Of Payments basis in tables C1 to C3 inclusive, and D1 to D3 in-

clusive, of the Monthly Review of External Trade Statistics. 
15.5 Price index numbers, by commodity group'

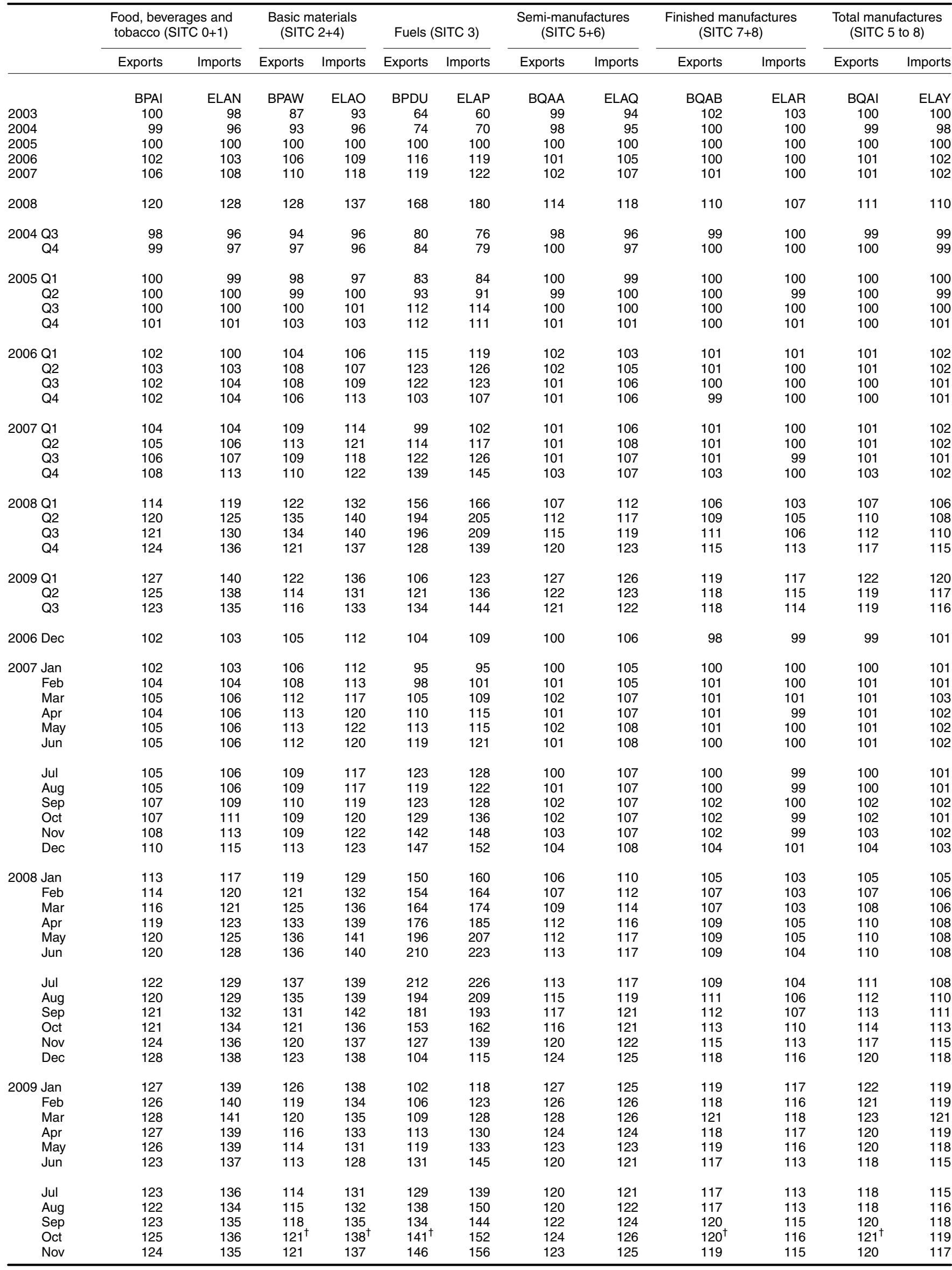

1 Commodity price indices are shown in more detail on a not seasonally adjusted Balance of Payments basis in tables C4 to C6 inclusive, and D4 to

D6 inclusive, of the Monthly Review of External Trade Statistics. 


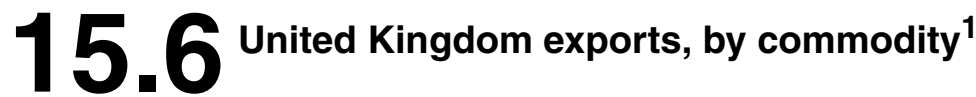

$£$ million Balance of Payments-consistent basis seasonally adjusted

\begin{tabular}{|c|c|c|c|c|c|c|c|c|c|c|c|c|}
\hline & & 2007 & 2008 & $\begin{array}{r}2009 \\
\text { Q1 }\end{array}$ & $\begin{array}{r}2009 \\
\text { Q2 }\end{array}$ & $\begin{array}{r}2009 \\
\text { Q3 }\end{array}$ & $\begin{array}{r}2009 \\
\text { Jun }\end{array}$ & $\begin{array}{r}2009 \\
\text { Jul }\end{array}$ & $\begin{array}{r}2009 \\
\text { Aug }\end{array}$ & $\begin{array}{r}2009 \\
\text { Sep }\end{array}$ & $\begin{array}{r}2009 \\
\text { Oct }\end{array}$ & $\begin{array}{r}2009 \\
\text { Nov }\end{array}$ \\
\hline 0. Food and live animals & BOGG & 7374 & 8681 & 2310 & 2297 & 2265 & 761 & 734 & 756 & 775 & $763^{\dagger}$ & \\
\hline \multicolumn{13}{|l|}{ Of which: } \\
\hline 01. Meat and meat preparations & BOGS & 839 & 1165 & 309 & 316 & 332 & 104 & 97 & 111 & 124 & $109^{\dagger}$ & \\
\hline 02. Dairy products and eggs & BQMS & 807 & 885 & 206 & 198 & 209 & 65 & 66 & 69 & 74 & $76_{+}^{\dagger}$ & \\
\hline $04 \& 08$. Cereals and animal feeding stuffs & BQMT & 1791 & 2283 & 617 & 614 & 576 & 209 & 195 & 191 & 190 & $188^{\dagger}$ & \\
\hline 1. Beverages and tobacco & BQMZ & 4395 & 5027 & 1242 & 1337 & 1343 & 453 & 428 & 446 & 469 & $465^{\dagger}$ & \\
\hline 11. Beverages & BQNB & 4093 & 4580 & 1133 & 1234 & 1249 & 422 & 397 & 415 & 437 & $432^{\dagger}$ & \\
\hline 12. Tobacco & BQOW & 302 & 447 & 109 & 103 & 94 & 31 & 31 & 31 & 32 & 33 & \\
\hline 2. Crude materials & $\mathrm{BQOX}$ & 5196 & 6266 & 1014 & 1150 & 1254 & 369 & 387 & 401 & 466 & $495^{\dagger}$ & \\
\hline
\end{tabular}

Of which:

24. Wood, lumber and cork
25. Pulp and waste paper
26. Textile fibres
28. Metal ores

3. Fuels

33. Petroleum and petroleum products

32, 34 and 35 . Coal, gas and electricity

\section{Animal and vegetable oils and fats}

5. Chemicals

Of which:

$$
\begin{aligned}
& \text { 51. Organic chemicals } \\
& \text { 52. Inorganic chemicals } \\
& \text { 53. Colouring materials } \\
& \text { 54. Medicinal products } \\
& \text { 55. Toilet preparations } \\
& \text { 57 \& 58. Plastics }
\end{aligned}
$$

6. Manufactures classified chiefly by material

Of which:

$$
\begin{aligned}
& \text { 63. Wood and cork manufactures } \\
& \text { 64. Paper and paperboard manufactures } \\
& \text { 65. Textile manufactures } \\
& \text { 67. Iron and steel } \\
& \text { 68. Non-ferrous metals } \\
& \text { 69. Metal manufactures }
\end{aligned}
$$

7. Machinery and transport equipment ${ }^{2}$

$71-716,72,73 \& 74$. Mechanical machinery

$716,75,76$ \& 77 . Electrical machinery

78. Road vehicles

79. Other transport equipment

8. Miscellaneous manufactures ${ }^{2}$

Of which:

84. Clothing
85. Footwear
87 \& 88. Scientific and photographic

9. Other commodities and transactions
BQOY

$\mathrm{BQOZ}$

$B O P A$

$B Q P B$

BOPN

ELBL

BOQI

BQPI

ENDG

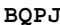

BQPK

CSCE

BQPL

CSCF

BQQA

BQQB

BQQC

BQQD

$B Q Q E$

$B Q Q F$

$\mathrm{BOOG}$

$\mathrm{BQQH}$

BQQI

BQQK

BQQL

BQQM

$B O O N$

BQQO $\begin{array}{rr}144 & 126 \\ 417 & 481 \\ 499 & 543 \\ 2898 & 3665\end{array}$

$24700 \quad 35684$

22756

1944

32145

3539

327

360

38891

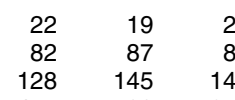

21
88
147

6
29
47

$$
\begin{array}{r}
7 \\
32 \\
50
\end{array}
$$

$$
\begin{array}{r}
7 \\
25 \\
45
\end{array}
$$

$\begin{array}{rrr}7 & 8^{\dagger} & 7 \\ 31 & 35 & 30 \\ 52 & 53 & 55\end{array}$

$6241 \quad 6493 \quad 6425 \quad 2200$

2232

$5447 \quad 5880$

6001

2010

267

$281^{\dagger} \quad 236$

$20492144 \quad 2428^{\dagger} 2490$

$\begin{array}{lll}794 & 613 \quad 424\end{array}$

190

160

$\begin{array}{llll}1930 & 1999 & 2241^{\dagger} & 2291\end{array}$

141

94

76

23

26

23

$27 \quad 28^{\dagger} \quad 22$

$\begin{array}{llllll}7601 & 8389 & 2044 & 1909 & 2710 & 731\end{array}$

$\begin{array}{rrrrrrrrrrr}7601 & 8389 & 2044 & 1909 & 2710 & 731 & 823 & 978 & 909 & 839^{\dagger} & 913 \\ 2830 & 2983 & 888 & 621 & 709 & 199 & 247 & 214 & 248 & 235^{\dagger} & 230 \\ 1672 & 1837 & 402 & 408 & 426 & 136 & 141 & 137 & 148 & 153^{\dagger} & 150 \\ 14507 & 17228 & 5090 & 4950 & 5081 & 1603 & 1648 & 1689 & 1744 & 1738^{\dagger} & 1769 \\ 3689 & 3945 & 1006 & 991 & 1052 & 330 & 345 & 351 & 356 & 363^{\dagger} & 351 \\ 4612 & 4859 & 1075 & 1098 & 1103 & 355 & 374 & 354 & 375 & 384^{\dagger} & 389 \\ & & & & & & & & & & \\ 29378 & 32394 & 6185 & 6055 & 6094 & 1980 & 1993 & 1993 & 2108 & 2209^{\dagger} & 2142\end{array}$

$\begin{array}{lllllllllll}29378 & 32394 & 6185 & 6055 & 6094 & 1980 & 1993 & 1993 & 2108 & 2209^{\dagger} & 2142\end{array}$

$\begin{array}{rrrrr}272 & 243 & 56 & 53 & 57 \\ 2124 & 2335 & 570 & 556 & 562 \\ 2589 & 2591 & 570 & 577 & 601 \\ 6016 & 6853 & 1289 & 1029 & 1087 \\ 5778 & 6866 & 949 & 957 & 1039\end{array}$

17
183
185
321
314
349

$\begin{array}{rrrrr}17 & 23 & 17 & 17^{\dagger} & 17 \\ 186 & 190 & 186 & 193^{\dagger} & 213 \\ 194 & 208 & 199 & 209^{\dagger} & 201 \\ 321 & 358 & 408 & 420^{\dagger} & 391 \\ 351 & 340 & 348 & 381^{\dagger} & 369 \\ 362 & 330 & 332 & 373^{\dagger} & 348\end{array}$

$\begin{array}{lllllllllll}82713 & 89189 & 19733 & 19110 & 19857 & 6336 & 6560 & 6444 & 6853 & 7243^{\dagger} & 7076\end{array}$

$\begin{array}{lllllllllll}28969 & 32273 & 7557 & 7426 & 7156 & 2393 & 2385 & 2344 & 2427 & 2492^{\dagger} & 2472\end{array}$

$\begin{array}{lllllllllll}24215 & 25280 & 6233 & 5812 & 6014 & 1859 & 1952 & 1994 & 2068 & 2235^{\dagger} & 2079\end{array}$

$\begin{array}{lllllllllll}21114 & 22477 & 3702 & 3671 & 4484 & 1321 & 1481 & 1487 & 1516 & 1768^{\dagger} & 1777\end{array}$

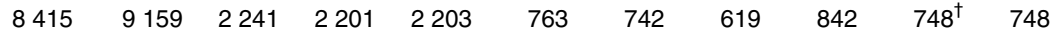

$\begin{array}{lllllllllll}26695 & 28468 & 6902 & 6814 & 6908 & 2266 & 2366 & 2252 & 2290 & 2264^{\dagger} & 2411\end{array}$ 


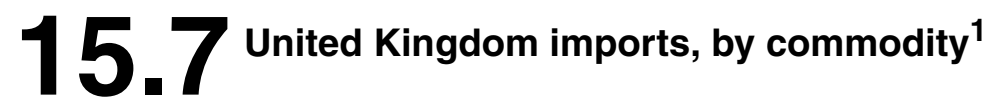

$£$ million Balance of Payments-consistent basis seasonally adjusted

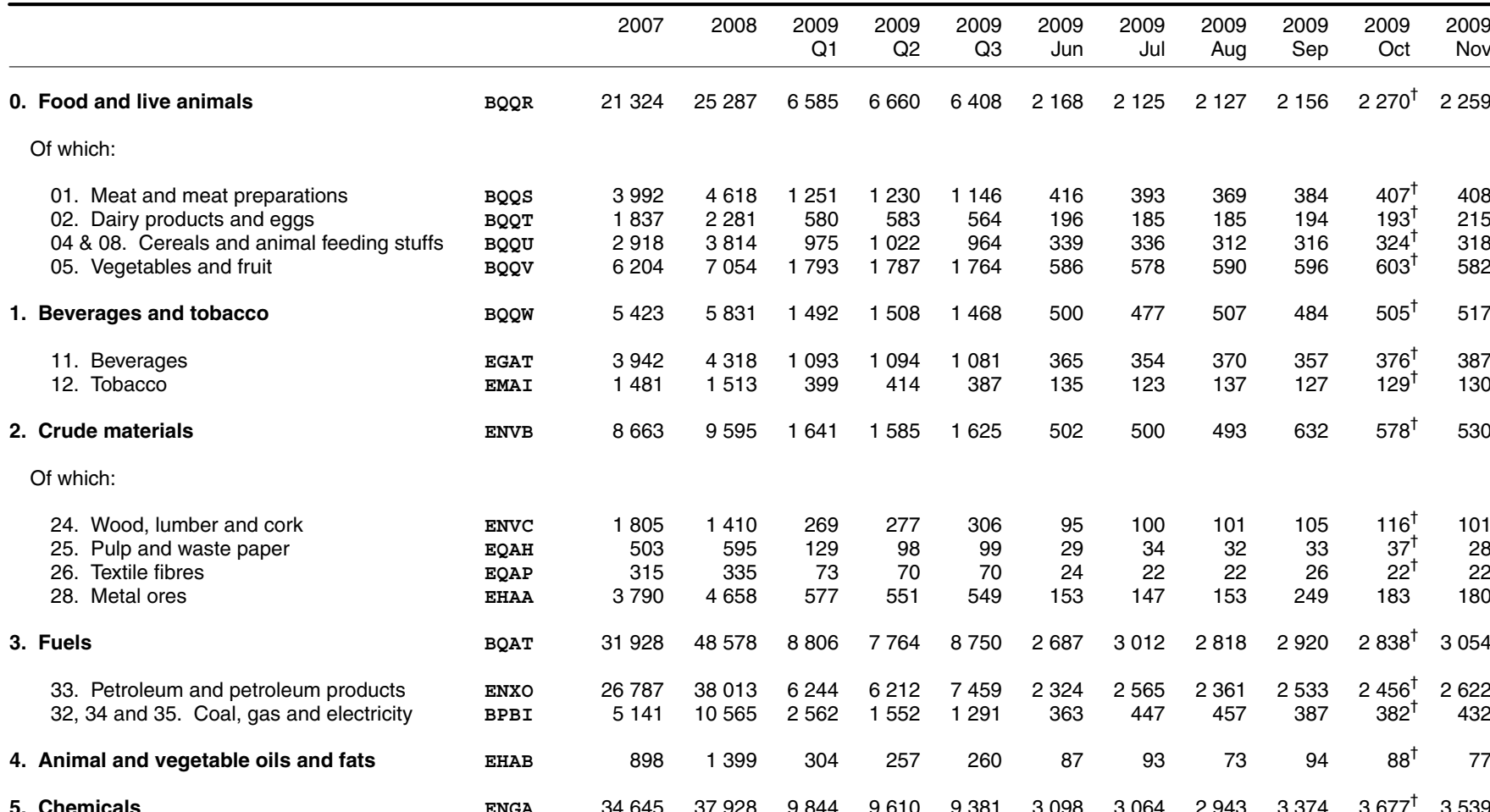

Of which:
51. Organic chemicals
52. Inorganic chemicals
53. Colouring materials
54. Medicinal products
55. Toilet preparations
57 \& 58. Plastics

6. Manufactures classified chiefly by material

Of which:

63. Wood and cork manufactures

64. Paper and paperboard manufactures

65. Textile manufactures

67. Iron and steel

68. Non-ferrous metals

69. Metal manufactures

7. Machinery and transport equipment ${ }^{2}$

$71-716,72,73 \& 74$. Mechanical machinery

$716,75,76$ \& 77 . Electrical machinery

78. Road vehicles

79. Other transport equipment

8. Miscellaneous manufactures ${ }^{2}$

Of which:

84. Clothing
85. Footwear
87 \& 88. Scientific and photographic

9. Other commodities and transactions
EHAI

EHAJ

EHAK

EHAT

EHAM

EHAN

EHAO

EHAQ

EHAR

EHAS

EHAT

EHAU

$\begin{array}{rrrrrrrrrrr}8620 & 8460 & 2070 & 2148 & 1886 & 630 & 510 & 527 & 849 & 792^{\dagger} & 810 \\ 2679 & 2756 & 780 & 610 & 556 & 173 & 205 & 171 & 180 & 377^{\dagger} & 217 \\ 1164 & 1228 & 277 & 291 & 294 & 100 & 99 & 96 & 99 & 97^{\dagger} & 97 \\ 9943 & 11049 & 3350 & 3286 & 3186 & 1112 & 1124 & 991 & 1071 & 1163^{\dagger} & 1209 \\ 3448 & 3915 & 1034 & 1034 & 1064 & 342 & 348 & 362 & 354 & 354^{\dagger} & 364 \\ 5699 & 6219 & 1305 & 1310 & 1425 & 440 & 458 & 470 & 497 & 563^{\dagger} & 521 \\ 39792 & 41910 & 8839 & 8861 & 8688 & 2761 & 2977 & 2778 & 2933 & 3044^{\dagger} & 3144\end{array}$

$\begin{array}{llllll}1733 & 1731 & 369 & 376 & 364 & 123\end{array}$

$\begin{array}{llrrr}5248 & 5492 & 1357 & 1347 & 1386\end{array}$

$\begin{array}{lllll}4084 & 4073 & 953 & 932 & 953\end{array}$

$\begin{array}{lllll}5958 & 6575 & 1029 & 884 & 908\end{array}$

$\begin{array}{llllll}6230 & 6419 & 1432 & 1534 & 1526 & 475\end{array}$

$\begin{array}{llllll}6563 & 6985 & 1569 & 1459 & 1433 & 462\end{array}$

$\begin{array}{llllll}117726 & 121009 & 25648 & 25284 & 27154 & 8603\end{array}$

$\begin{array}{lllllllllll}25776 & 28916 & 6315 & 5955 & 5930 & 1978 & 1940 & 1927 & 2063 & 1970^{\dagger} & 2047\end{array}$

$\begin{array}{llllllllllll}46006 & 47588 & 10995 & 10638 & 11000 & 3515 & 3551 & 3698 & 3751 & 4019^{\dagger} & 3941\end{array}$

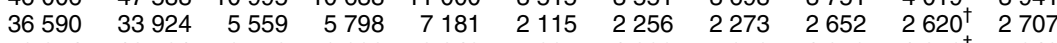

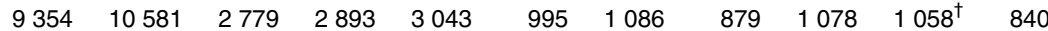

$\begin{array}{lllllllllll}47939 & 50921 & 12887 & 12424 & 12179 & 4054 & 4090 & 4082 & 4007 & 4239^{\dagger} & 4067\end{array}$

\begin{tabular}{|c|c|c|c|c|c|c|c|c|c|c|}
\hline CSDR & 12310 & 13210 & 3620 & 3527 & 3458 & 1150 & 1185 & 1178 & 1095 & $1165^{\dagger}$ \\
\hline CSDS & 2659 & 2840 & 794 & 784 & 780 & 255 & 266 & 259 & 255 & $269^{\dagger}$ \\
\hline HAW & 7572 & 8443 & 2216 & 2079 & 2072 & 674 & 695 & 690 & 687 & $728^{\dagger}$ \\
\hline
\end{tabular}

BOKH

$\begin{array}{lllllllllll}310612 & 345024 & 76654 & 74583 & 76589 & 24669 & 25390 & 24807 & 26392 & 27 & 189^{\dagger} 26978\end{array}$

1 The numbers on the left hand side of the table refer to the code numbers of 2 Sections 7 and 8 are shown by broad economic category in table G2 of the the Standard International Trade Classification, Revision 3, which was intro- $\quad$ Monthly Review of External Trade Statistics.

duced in January 1988. 
450 United Kingdom exports, by area

$£$ million Balance of Payments-consistent basis seasonally adjusted

\begin{tabular}{|c|c|c|c|c|c|c|c|c|c|c|c|c|}
\hline & & 2007 & 2008 & $\begin{array}{r}2009 \\
\text { Q1 }\end{array}$ & $\begin{array}{r}2009 \\
\text { Q2 }\end{array}$ & $\begin{array}{r}2009 \\
\text { Q3 }\end{array}$ & $\begin{array}{r}2009 \\
\text { Jun }\end{array}$ & $\begin{array}{r}2009 \\
\text { Jul }\end{array}$ & $\begin{array}{r}2009 \\
\text { Aug }\end{array}$ & $\begin{array}{r}2009 \\
\text { Sep }\end{array}$ & $\begin{array}{r}2009 \\
\text { Oct }\end{array}$ & $\begin{array}{r}2009 \\
\text { Nov }\end{array}$ \\
\hline European Union: ${ }^{1}$ & LGCK & 127813 & 141428 & 30875 & 29556 & 31418 & 9992 & 10351 & 10178 & 10889 & $11092^{\dagger}$ & 1123 \\
\hline \multicolumn{13}{|l|}{ of which: } \\
\hline Belgium \& Luxembourg & CHNQ & 12122 & 13579 & 2939 & 2813 & 2699 & 928 & 919 & 882 & 898 & $953^{\dagger}$ & 100 \\
\hline Cyprus & BQGN & 415 & 536 & 119 & 166 & 192 & 79 & 58 & 69 & 65 & $49_{+}^{\dagger}$ & \\
\hline France & ENYL & 18103 & 18117 & 4158 & 3951 & 4426 & 1350 & 1534 & 1373 & 1519 & $1517_{+}^{\dagger}$ & 155 \\
\hline Germany & ENYO & 24699 & 27890 & 6001 & 5765 & 6224 & 1887 & 2088 & 1976 & 2160 & $2217^{\dagger}$ & 226 \\
\hline Greece & CHNT & 1350 & 1661 & 386 & 370 & 450 & 132 & 148 & 134 & 168 & 141 & \\
\hline Irish Republic & CHNS & 17801 & 19069 & 4015 & 3925 & 3857 & 1244 & 1209 & 1276 & 1372 & $1316_{+}^{\dagger}$ & 133 \\
\hline Italy & CHNO & 9189 & 9370 & 2136 & 1998 & 2092 & 684 & 694 & 672 & 726 & $707_{+}^{\dagger}$ & \\
\hline Malta & BQGY & 362 & 449 & 96 & 94 & 92 & 31 & 28 & 29 & 35 & $24_{+}^{\dagger}$ & \\
\hline Netherlands & CHNP & 15115 & 19849 & 4451 & 4130 & 4584 & 1452 & 1469 & 1538 & 1577 & $1711^{\dagger}$ & 171 \\
\hline Portugal & CHNU & 1481 & 1636 & 389 & 391 & 351 & 125 & 107 & 119 & 125 & $127_{+}^{\dagger}$ & 12 \\
\hline Slovakia & $\mathrm{BQHB}$ & 382 & 457 & 86 & 83 & 103 & 29 & 28 & 41 & 34 & $40_{+}^{\dagger}$ & \\
\hline Slovenia & BQHE & 205 & 224 & 45 & 41 & 41 & 17 & 14 & 12 & 15 & $16_{+}^{\dagger}$ & \\
\hline Spain & CHNV & 9979 & 10202 & 2243 & 2113 & 2327 & 741 & 766 & 768 & 793 & $878^{\dagger}$ & \\
\hline \multicolumn{13}{|l|}{ Of which: } \\
\hline Bulgaria & WYUF & 202 & 253 & 61 & 47 & 46 & 16 & 16 & 15 & 15 & $15^{\dagger}$ & \\
\hline Czech Rep & FKML & 1401 & 1544 & 342 & 337 & 373 & 117 & 118 & 119 & 136 & $137^{\dagger}$ & \\
\hline Denmark & CHNR & 2182 & 2584 & 576 & 574 & 652 & 197 & 227 & 182 & 243 & 211 & \\
\hline Estonia & AUEV & 228 & 220 & 32 & 30 & 38 & 12 & 9 & 10 & 19 & $19^{\dagger}$ & \\
\hline Hungary & QALC & 863 & 1008 & 217 & 215 & 215 & 65 & 65 & 80 & 70 & 71 & \\
\hline Latvia & BQGQ & 145 & 169 & 32 & 25 & 24 & 7 & 7 & 8 & 9 & 11 & \\
\hline Lithuania & BQGU & 311 & 282 & 46 & 40 & 42 & 14 & 12 & 12 & 18 & 15 & \\
\hline Poland & ERDR & 2372 & 3004 & 644 & 699 & 710 & 281 & 218 & 249 & 243 & $252_{+}^{\dagger}$ & \\
\hline Romania & WMDB & 668 & 755 & 168 & 166 & 170 & 53 & 54 & 59 & 57 & $69^{\dagger}$ & \\
\hline Sweden & CHNA & 4904 & 5198 & 1021 & 1005 & 1019 & 337 & 328 & 329 & 362 & $375^{\dagger}$ & \\
\hline Other Western Europe: & HCJD & 9232 & 10757 & 2292 & 2482 & 2287 & 765 & 785 & 728 & 774 & $863^{\dagger}$ & \\
\hline
\end{tabular}

Of which:

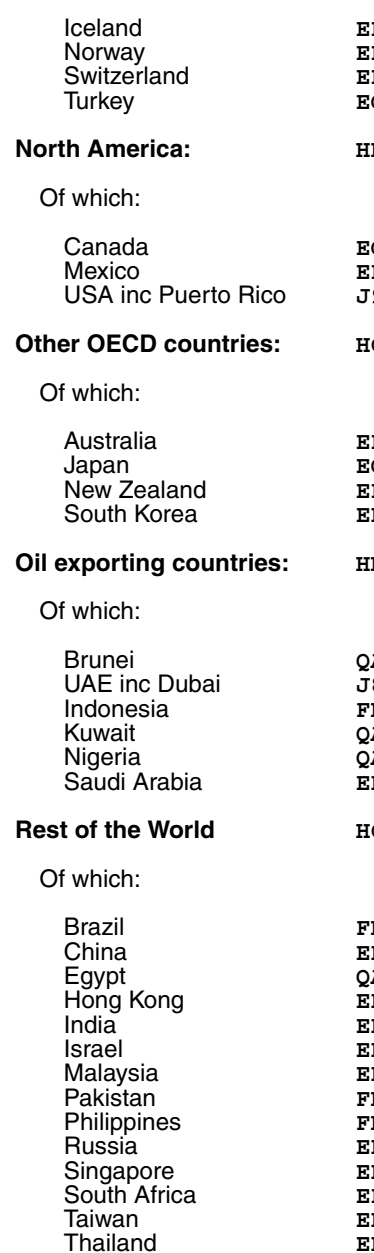

EPLW

EPLX

EOBA

HBZQ

EOBC

EPJX

J9C5

HCII

EPMA

EOBD

ERMB

HDII

QALF

J8YH

OATB

QATE

HCHW

FKMO
ERDN
QALL
ERDG
ERDJ
ERDL
ERDK
FKMU
FKMX
ERDQ
ERDH
EPME
ERDP
ERDO

198
2697

3808

187
2850
465

4656
2568

40
672

972
561

33
729
999
595

29
647
92

8
207

300

12
231
319
188

9
190

300

8
226

36365

39623

9722

8959

$9351 \quad 2992$

3054

3204

188

$\begin{array}{rr}10 & 8 \\ 270^{\dagger} & 262\end{array}$

$\begin{array}{ll}336^{\dagger} & 411 \\ 202^{\dagger} & 190\end{array}$

$3225^{\dagger} \quad 3293$

\section{$3291 \quad 326$ \\ $\begin{array}{rr}801 & 904 \\ 32274 & 35436\end{array}$}

842
191
8689

$\begin{array}{lll}812 & 788 & 241\end{array}$

240

263

285

$8778 \quad 9948$

2172

7948

$\begin{array}{rr}165 & 68 \\ 8398 & 2683\end{array}$

63
2751

2890

51
2757

$\begin{array}{rr}291^{\dagger} & 322 \\ 54^{\dagger} & 71\end{array}$

2136

$2286 \quad 703$

807

699

780

$822^{\dagger} \quad 822$

\section{$\begin{array}{ll}2630 & 3103 \\ 3866 & 3908\end{array}$ \\ 3866}

364
1914

3908
385

729
928
79
436

742
795
80

80
519

$\begin{array}{ll}745 & 248 \\ 899 & 262\end{array}$

999
546

248
262
166

$\begin{array}{rr}245 & 237 \\ 302 & 314\end{array}$

$\begin{array}{rr}42 & 26 \\ 218 & 122\end{array}$

263
283

283
286

$259^{\dagger} \quad 267$

$\begin{array}{cr}315^{\dagger} & 309 \\ 30 & 27\end{array}$

$\begin{array}{rr}30 & 27 \\ 218 & 219\end{array}$

$9716 \quad 11618$

2966

2766

$2713 \quad 883$

918

851

944

$964^{\dagger} \quad 1001$

1 Includes; Austria, Belgium, Bulgaria, Cyprus, Czech Republic, Denmark

Estonia, Finland, France, Germany, Greece, Hungary, Irish Republic, Italy,

$\begin{array}{rrrrrrr}1108 & 1694 & 368 & 403 & 490 & 131 & 148 \\ 3860 & 5084 & 1180 & 1276 & 1376 & 431 & 440 \\ 686 & 944 & 230 & 260 & 225 & 89 & 80 \\ 2726 & 3676 & 858 & 985 & 894 & 317 & 260 \\ 2968 & 4135 & 651 & 778 & 806 & 260 & 335 \\ 1257 & 1341 & 225 & 294 & 300 & 102 & 122 \\ 975 & 1135 & 228 & 259 & 279 & 93 & 85 \\ 423 & 475 & 130 & 125 & 105 & 38 & 36 \\ 251 & 245 & 63 & 65 & 72 & 22 & 23 \\ 2893 & 4275 & 671 & 602 & 505 & 172 & 171 \\ 2467 & 2820 & 709 & 762 & 736 & 249 & 246 \\ 2244 & 2658 & 547 & 513 & 533 & 170 & 162 \\ 957 & 892 & 195 & 180 & 199 & 59 & 67 \\ 613 & 757 & 150 & 199 & 244 & 72 & 77\end{array}$

\begin{tabular}{rrrrr}
148 & 167 & 175 & $188^{\dagger}$ & 135 \\
440 & 459 & 477 & $465^{\dagger}$ & 490 \\
80 & 81 & 64 & 83 & 99 \\
260 & 300 & 334 & $306^{\dagger}$ & 323 \\
335 & 224 & 247 & $228^{\dagger}$ & 212 \\
122 & 77 & 101 & 109 & 104 \\
85 & 99 & 95 & 104 & 82 \\
36 & 39 & 30 & 34 & 37 \\
23 & 23 & 26 & 23 & 24 \\
171 & 188 & 146 & $221^{\dagger}$ & 197 \\
246 & 243 & 247 & $255^{\dagger}$ & 208 \\
162 & 185 & 186 & $288^{\dagger}$ & 181 \\
67 & 62 & 70 & 71 & 62 \\
77 & 61 & 106 & 114 & 92 \\
\hline
\end{tabular}

Latvia, Lithuania, Luxembourg, Malta, Netherlands, Poland, Portugal, Romania, Slovakia, Slovenia, Spain, and Sweden. 
$£$ million Balance of Payments-consistent basis seasonally adjusted

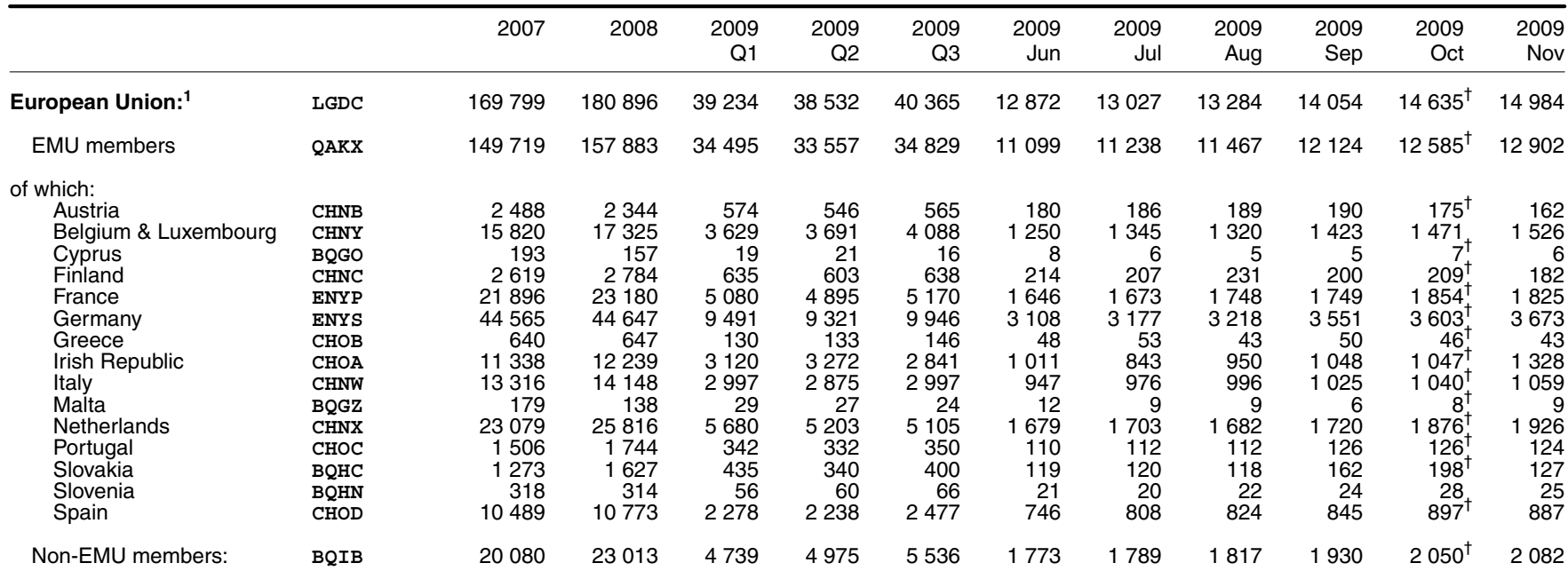

Of which:

Bulgaria
Czech Rep
Denmark
Estonia
Hungary
Latvia
Lithuania
Poland
Romania
Sweden

Other Western Europe:

Of which:

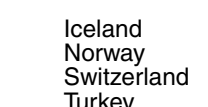

Turkey

North America:

Of which:

$$
\begin{aligned}
& \text { USA inc Puerto Rico } \\
& \text { Canada } \\
& \text { Mexico }
\end{aligned}
$$

Other OECD countries:

Of which:

Australia

Japan

New Zealand

South Korea

Oil exporting countries:

Of which:

Brunei
UAE inc Dubai
Indonesia
Kuwait
Nigeria
Saudi Arabia

Rest of the World

Of which:

$\begin{array}{ll}\text { Brazil } & \text { FKMP } \\ \text { China } & \text { ERDZ } \\ \text { Egypt } & \text { QALM } \\ \text { Hong Kong } & \text { ERDS } \\ \text { India } & \text { ERDV } \\ \text { Israel } & \text { ERDX } \\ \text { Malaysia } & \text { ERDW } \\ \text { Pakistan } & \text { FKMV } \\ \text { Philippines } & \text { FKMY } \\ \text { Russia } & \text { EREC } \\ \text { Singapore } & \text { ERDT } \\ \text { South Africa } & \text { EPNE } \\ \text { Taiwan } & \text { EREB } \\ \text { Thailand } & \text { EREA }\end{array}$

WYUT
FKMM
CHNZ
BQGL
QALD
BQGR
BQGV
ERED
WMDC
CHND

HDJQ

EPNB

239

2983
3444

3444
226

2377

605
299

3695

3695
938
5274

24359
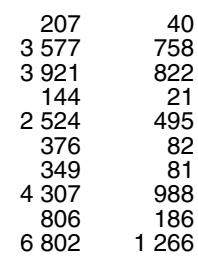

3577

144

2524
376

349
4307

6806

32436

40

40
803
873

873
32
516

516
83

88
1044
176

1320

6724

5985

415
14316
4746

4746
4632

458
21609

5256

114
4233

4262
11064

124
3399
1287
1120

121
4047
1346

32472

32627

8518

7291

1185

6780

43

961

535
62

62
95

1253
193

1413

6759

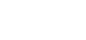

26095
5793
582

26009

703

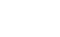

55

13870

5824
794

1298
188

1139
178

1017
209

28

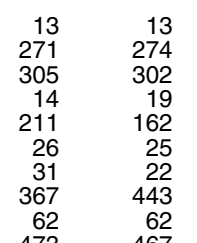

13
274
302
19
162
25
22
443
62
467

16
353
303
7
186
20
43
347
63
479

14
317
356
11
187
17
30
463
68
467

$\begin{array}{rr}16^{\dagger} & 16 \\ 311^{\dagger} & 322\end{array}$

$\begin{array}{ll}368^{\dagger} & 378 \\ 18 & 7\end{array}$

$189^{\dagger} \quad 226$

$\begin{array}{rr}189 & 226 \\ 23 & 22\end{array}$

$\begin{array}{rr}26 & 327^{\dagger} \\ 79^{\dagger} & 48\end{array}$

$\begin{array}{rr}79^{\dagger} & 70 \\ 493^{\dagger} & 524\end{array}$

2035

2203

2211

2345

$2179^{\dagger} \quad 2434$

15192

3327

3155

3079

1101

42
1183
418
375

36
1289
433

42
1360
404

43
1398

398
509
379

$\begin{array}{rr}39^{\dagger} & 45 \\ 1186^{\dagger} & 1513 \\ 470^{\dagger} & 468\end{array}$

$\begin{array}{lllll}475 & 370 & 410^{\dagger} & 391\end{array}$

2245

2
7885
667

2389
8546

670
1656

522

667
3073

748
3509

497
1727
1969

6387

7995

1656
218
783

1626

208
799

1727
196
659

$\begin{array}{rr}154 & 209 \\ 643 & 593 \\ 73 & 66\end{array}$

73
231

66
234

155
567
64

64
221

133
567
66

66
204

$\begin{array}{ll}152 & 231 \\ 533^{\dagger} & 507\end{array}$

$\begin{array}{rr}62 & 69 \\ 223 & 202\end{array}$

596

662

545

713

509

739

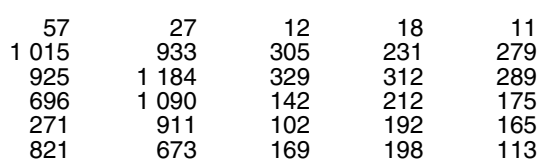

$\begin{array}{rr}- & 4 \\ 75 & 94 \\ 96 & 97 \\ 79 & 78 \\ 47 & 70 \\ 67 & 30\end{array}$

$\begin{array}{rr}4 & \\ 94 & 83 \\ 97 & 93 \\ 78 & 37 \\ 70 & \\ 30 & \end{array}$

$\begin{array}{rr}1 & 6 \\ 83 & 102 \\ 93 & 99 \\ 37 & 60 \\ 3 & 92 \\ 41 & 42\end{array}$

$\begin{array}{rr}1{ }^{\dagger} & 3 \\ 94^{\dagger} & 99 \\ 92^{\dagger} & 87 \\ 50 & 76 \\ 4 & 71 \\ 37 & 60\end{array}$

63725

$75878 \quad 17069$

17740

17686

5786

6053

$5614 \quad 6019 \quad 6270^{\dagger} \quad 5433$

$\begin{array}{rr}2061 & 2619 \\ 18734 & 23175 \\ 538 & 640 \\ 6939 & 8081 \\ 3809 & 4490 \\ 1045 & 1155 \\ 1684 & 1872 \\ 512 & 630 \\ 717 & 629 \\ 5248 & 6928 \\ 4247 & 4007 \\ 3060 & 4739 \\ 2418 & 2598 \\ 2012 & 2427\end{array}$

628
6038
167
1945
1138
270
447
173
111
1271
857
808
557
581

661
6103
190
1891
1093
272
417
158
108
1162
914
1070
522
574

$\begin{array}{rr}625 & 230 \\ 6060 & 1989 \\ 151 & 74 \\ 1913 & 632 \\ 1180 & 358 \\ 258 & 97 \\ 404 & 141 \\ 183 & 59 \\ 96 & 37 \\ 1076 & 441 \\ 790 & 228 \\ 970 & 327 \\ 552 & 174 \\ 561 & 200\end{array}$

$\begin{array}{rr}230 & 220 \\ 1989 & 2026 \\ 74 & \\ 632 & 636 \\ 358 & 394 \\ 97 & 87 \\ 141 & 141 \\ 59 & 56 \\ 37 & 38 \\ 441 & 397 \\ 228 & 237 \\ 327 & 432 \\ 174 & 182 \\ 200 & 184\end{array}$

$\begin{array}{rr}220 & 186 \\ 026 & 2027 \\ 70 & 33 \\ 636 & 635 \\ 394 & 391 \\ 87 & 84 \\ 141 & 130 \\ 56 & 64 \\ 38 & 31 \\ 397 & 297 \\ 237 & 244 \\ 432 & 146 \\ 182 & 185 \\ 184 & 189\end{array}$

$\begin{array}{rr}186 & 219 \\ 027 & 2007 \\ 33 & 48 \\ 635 & 642 \\ 391 & 395 \\ 84 & 87 \\ 130 & 133 \\ 64 & 63 \\ 31 & 27 \\ 297 & 382 \\ 244 & 309 \\ 146 & 392 \\ 185 & 185 \\ 189 & 188\end{array}$

$\begin{array}{rr}201^{\dagger} & 178 \\ 2190^{\dagger} & 1985 \\ 67 & 55 \\ 644^{\dagger} & 600 \\ 382^{\dagger} & 365 \\ 97 & 92 \\ 126^{\dagger} & 119 \\ 63 & 58 \\ 31 & 25 \\ 366 & 271 \\ 427 & 304 \\ 362 & 290 \\ 183^{\dagger} & 196 \\ 191^{\dagger} & 163\end{array}$

Source: Office for National Statistics: 01633456294

Includes: Austria, Belgium, Bulgaria, Cyprus, Czech Republic, Denmark,

Estonia, Finland, France, Germany, Greece, Hungary, Irish Republic, Italy,

Latvia, Lithuania, Luxembourg, Malta, Netherlands, Poland, Portugal, Ro-

mania, Slovakia, Slovenia, Spain, and Sweden. 